\title{
Temperature Rise in Superfluid Helium Pumps
}

\section{Peter Kittel}

\author{
Gerengy \\ silf? ? 6198 R \\ LARULE RESEARCH CENTER \\ IIMMON. Vingind
}

July 1988 
NASA Technical Memorandum 100997

\section{Temperature Rise in Superfluid Helium Pumps}

Peter Kittel, Ames Research Center, Moffett Field, California

July 1988

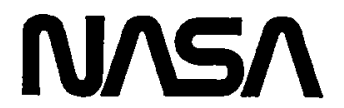

National Aeronautics and

Space Administration 
TEMPERATURE RISE IN SUPERFLUID HELIUM PUMPS

Peter Kittel

NASA Ames Research Center, Moffett Field, California, U.S.A.

The temperature rise of a fountain effect pump (FEP) and of a centrifugal pump (CP) are compared. Calculations and estimates presented here show that under the operating conditions expected during the resupply of superfluid helium in space, a centrifugal pump will produce a smaller temperature rise than will a fountain effect pump. The temperature rise for the FEP is calculated assuming an ideal pump, while the temperature rise of the $\mathrm{CP}$ is estimated from the measured performance of a prototype pump. As a result of this smaller temperature rise and of the different operating characteristics of the two types of pumps, transfers will be more effective using a centrifugal pump.

The temperature rise in a superfluid helium pump is an important factor in the design of a liquid helium resupply system for use in space. 1,2 This temperature rise signiflcantly affects losses during helium transfers and is one of the determining factors in the selection of the temperature of the supply tank. Here the temperature rise of a fountain-effect pump (FEP) and of a centrifugal pump (CP) are compared. Also, the operating characteristics of the pumps (FEP operating at constant heat input and the CP operating at constant speed) are discussed in terms of the operational requirements of helium resupply.

The FEP is assumed to be ideal. This is a good assumption based on the recent test results of the ceramic material that is currently planned for use in the Superfluid Helium On-Orbit Transfer (SHOOT) project.3,4 Discussions on non-ideal flow through FEPs have been given elsewhere. 5,6 However, in those discussions only the operating characteristics were considered, not the effects of the temperature rise. The operating mode being considered is that of operating the pump at constant heat input. The preceding assumptions result in the temperature rise being independent of flow, and thereby dependent only on the supply temperature and pressure rise:

$$
\Delta P=\int_{T}^{T+\Delta T} \rho S d T
$$

where $\Delta P$ is the pressure $r$ ise, $T$ is the inlet temperature, $\Delta T$ is the temperature rise, $\rho$ is the density, and $S$ is the entropy. Equation (1) is the thermomechanical effect. Combining this with the mechanocaloric effect,

$$
\dot{Q}=\dot{m} S T
$$

where $\dot{Q}$ is the heat inpyt and $\dot{\text { in }}$ is the mass flow, results in a constant-heat operating characteristic: 6

$$
\Delta P=\left(22 \dot{Q} / \dot{\mathrm{m}}-428 \mathrm{~T}^{6.6}\right)
$$


and

$$
\Delta T=\left(2.34 \times 10^{-3} \Delta P+T^{6.6}\right)^{0.152}-T
$$

where it has been assumed that $S \approx 19.5 \mathrm{~T}^{5.6}$ and $\rho \approx 145 \mathrm{~kg} \cdot \mathrm{m}^{-3}$. For an FEP operating at constant heat input, equation (3) gives the $\Delta P$ versus $\dot{m}$ operating characteristic. Equation (4) states that $\Delta T$ is a function of $\Delta P$ and $T$ only. These are shown in figure 1 , where the temperature rise for selected $\Delta P^{\prime} S$ and selected constant-Q operating curves are shown.

For the CP, an upper limit of the temperature rise is calculated from the measured efficiencies of the pump and motor and from the measured operating characteristics. The temperature rise is given by

$$
\Delta T \leq \frac{\grave{Q}_{p}}{\dot{m} C_{P}}+\left[\frac{d T}{d P}\right]_{S} \Delta P
$$

where $\dot{Q}_{p}$ is the heat dissipated in the pump and $C_{p}$ is the specific heat of the liquid. The second term on the right-hand side of equation (5L is due to the adiabatic (1deal) compression of the liquid by the pump; it is small and can be neglected. The inequality in equation (5) comes from assuming that all of $\dot{Q}_{p}$ goes into heating the liquid at the outlet of the pump and that none of it is conducted back to the supply tank. The heat input $\dot{Q}_{p}$ is given by

$$
\dot{Q}_{p}=n_{m}\left(1-n_{P}\right) \dot{Q}
$$

where $\eta_{m}$ is the motor efficiency, $\eta_{P}$ is the pump efficiency, and $\dot{Q}$ is the input power of the motor (fig. 2).

Using the correlations of Steward, ${ }^{8}$ this upper temperature limit can be found

$$
\Delta T=\left(4.18 \times 10^{-4} \Delta P \frac{1-n_{P}}{{ }^{n} P}+T^{6.6}\right) 0.152-T
$$

where the pump efficiency is

$$
\begin{aligned}
\eta_{P}= & {\left[2.68 \times 10^{5}\left(\frac{\dot{m}}{\mathrm{~N}}\right)-4.51 \times 10^{10}\left(\frac{\dot{\mathrm{m}}}{\mathrm{N}}\right)+1.69 \times 10^{15}\left(\frac{\dot{\mathrm{m}}}{\mathrm{N}}\right)^{3}\right] } \\
& \times\left(\frac{\mathrm{N}}{7 \times 10^{3}}\right)\left(2-\frac{\mathrm{N}}{7 \times 10^{3}}\right)
\end{aligned}
$$

and the pump speed (rpm) is

$$
\mathrm{N}=6.8 \times 10^{3} \dot{\mathrm{m}}+\left(1.1 \times 10^{10} \dot{\mathrm{m}}^{2}+2.16 \times 10^{3} \Delta \mathrm{P}\right)^{1 / 2}
$$

Equation (7c) is the operating characteristic of a pump operating at constant speed; equations (7a) and (7c) are shown in figure 1.

A comparison of equations (1) and (7a) reveals four principal differences between CP's and FEP's. First, the temperature rise across the $C P$ is lower than the rise across the FEP except for very low flows and high pressure rises. Thus, under normal operating conditions the $\mathrm{CP}$ will produce a lower temperature rise than will the 
FEP. As a result, the transfer effectiveness will be higher for the CP. ${ }^{2}$ Only briefly during the cooldown of the transfer line or during the early stages of cooling a warm Dewar, will the FEP have a higher transfer effectiveness. Overall, the CP would result in a more effective system.

Second, the outlet temperature of the FEP at high pressure rises is only slightly dependent on the inlet temperature. Thus, under cooldown conditions the effectiveness cannot be significantly improved by lowering the supply temperature. Under lowpressure-rise conditions, the effectiveness can be dramatically improved by lowering the supply temperature for an FEP system. The effectiveness of a CP system is less dependent on temperature. This may simplify the construction of a resupply system that uses a CP. Smaller vent lines (with a corresponding smaller parasitic heat load) could be used.

Third, under the conditions expected during the cooling down of a warm recelving tank (low flow, high pressure, and a system whose impedance decreases with time) the CP has a better operating characteristic than the FEP. This can be seen by comparing the slopes of the operating characteristic $a_{F E P}=-(d \Delta P / d \dot{m})_{Q}$ for the FEP operating at constant $\dot{Q}$ and $a_{C P}=-(d \Delta P / d \dot{m})_{r p m}$ for the CP operating at constant speed. From equations (3) and (7c) we find, respectively, $\alpha_{\mathrm{FEP}}=22 \dot{\mathrm{m}}^{-2}$ and $\alpha_{\mathrm{CP}} \approx 10^{7} \dot{\mathrm{m}}$. Under the above conditions $\left(\dot{\mathrm{m}}-4 \times 10^{-3} \mathrm{~kg} / \mathrm{s}\right), a_{\mathrm{FEP}} \gg \alpha_{\mathrm{CP}}$. Thus, the output of the FEP will drop rapidly with time because of the changing impedance, whereas the output of the CP will not. In practical terms, if near-constant mass flow is wanted, then a feedback control system may be required to keep the output of the FEP constant with time. A CP would not require such a control system.

And fourth, for a $C P$ there is an optimum operating curve ( $\triangle P$ vs $\dot{m}$ ) for which the temperature rise is minimized. This optimum is near the curve of maximum thermodynamic efficiency. ${ }^{8}$ For an optimized (maximum transfer effectiveness) system, the impedance of the transfer line should be designed to roughly follow the curve of minimum temperature rise.

In summary, under most superfluid operating conditions a centrifugal pump will produce a smaller temperature rise than will a fountain-effect pump. As a result transfers will be more effective using a centrifugal pump.

\section{REFERENCES}

1 Kittel, P. 'Orbital Resupply of Liquid Helium'. J. Spacecraft and Rockets, vol. 23 (1986), p. 391.

2 Kittel, P. 'Liquid Helium Pumps for In-Orbit Transfer'. Cryogenics, vol. 27 (1987), p. 81 .

3 DiPirro, M. J. and Kittel, P. 'Superfluid Helium On-Orbit Transfer (SHOOT)', Advances in Cryogenic Engineering, vol. 33 (1988), p. 893.

4 DiPirro, M. J. 'Superfluid Hellum On-Orbit Transfer Flight Experiment: Performance Estimates'. Cryogenics, vol. 28 (1988), p. 77.

5 Kittel, P. 'Losses in Fountain Effect Pumps'. Proceedings of the Eleventh International Cryogenic Engineering Conference, Butterworth, Guildford, UK (1986), p. 317 . 
6 Kittel, P. 'Operation Characteristics of Isocaloric Fountain-Effect Pumps'. Advances in Cryogenic Engineering, vol. 33 (1988), p. 465.

7 London, F. Superfluids, Vol. 2, Wiley, New York (1954).

8 Steward, W. G. 'A Centrifugal Pump for Liquid Helium'. Cryogenics, vol. 26 (1986), p. 103.
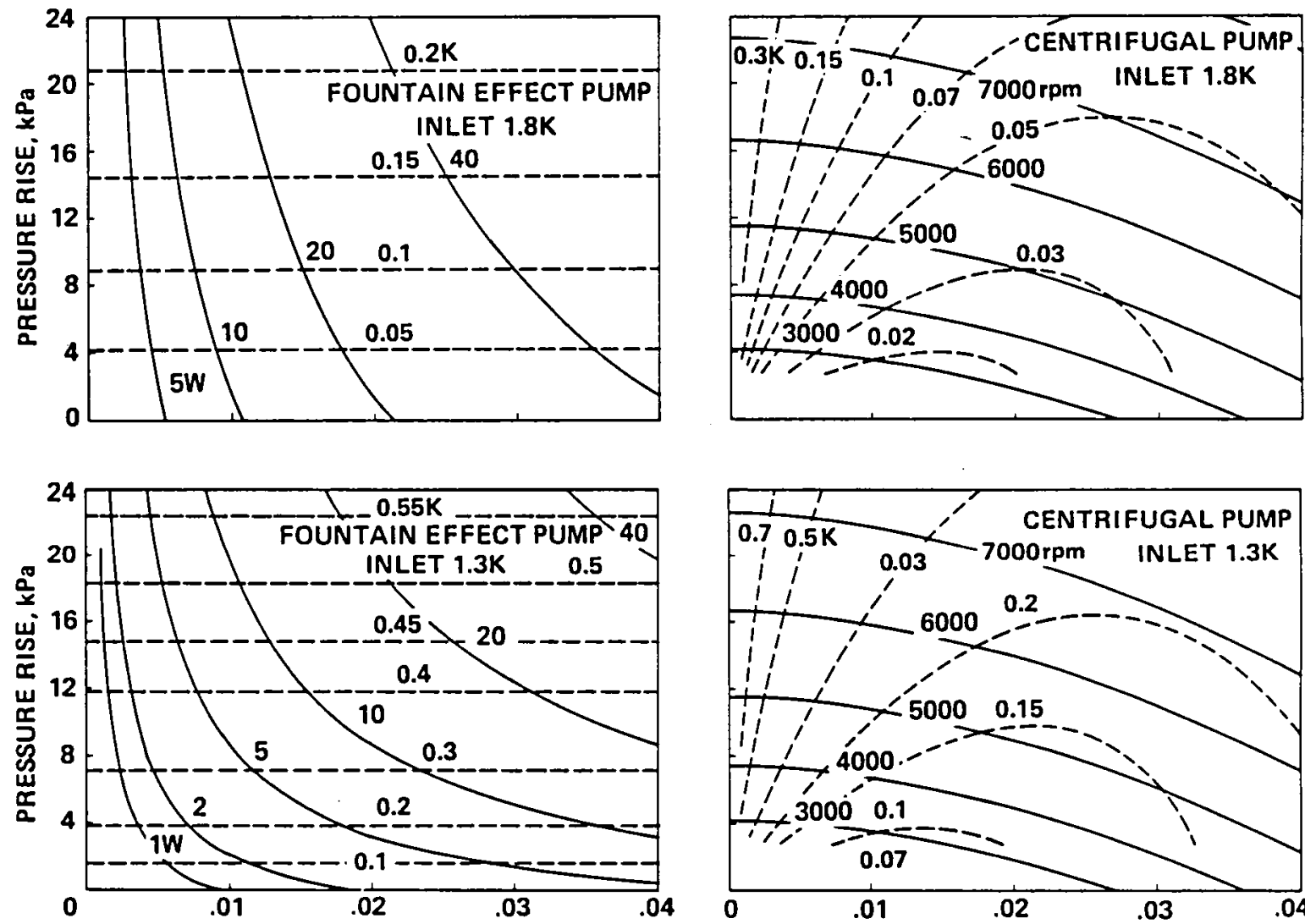

(a)

FLOW, $\mathrm{kg} / \mathrm{s}$

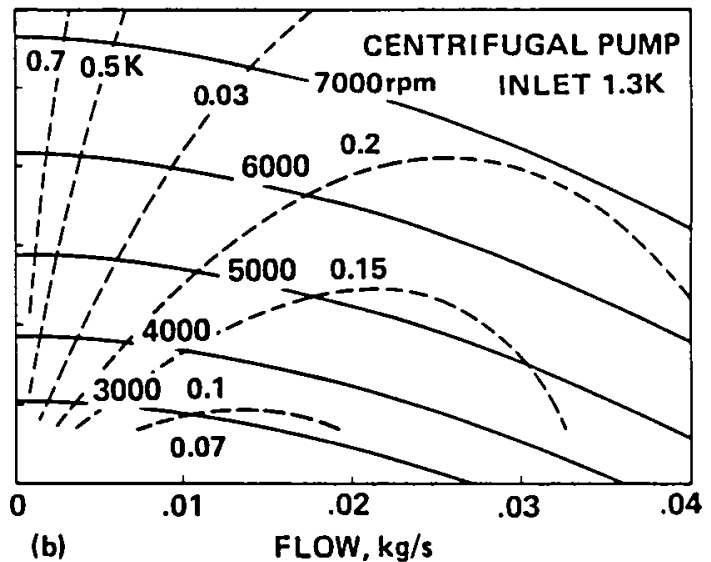

Fig. 1 Pressure rise versus flow for a fountain-effect pump (a) and centrifugal pump (b) for inlet temperatures of $1.8 \mathrm{~K}$ and $1.3 \mathrm{~K}$. The solid lines are curves of constant power input (watts) for the FEP and curves of constant speed (rpm) for the $\mathrm{CP}$. The dashed lines are curves of constant temperature rise (kelvin). In the case of the CP temperature rise, the curves are upper limits of the temperature increase. 


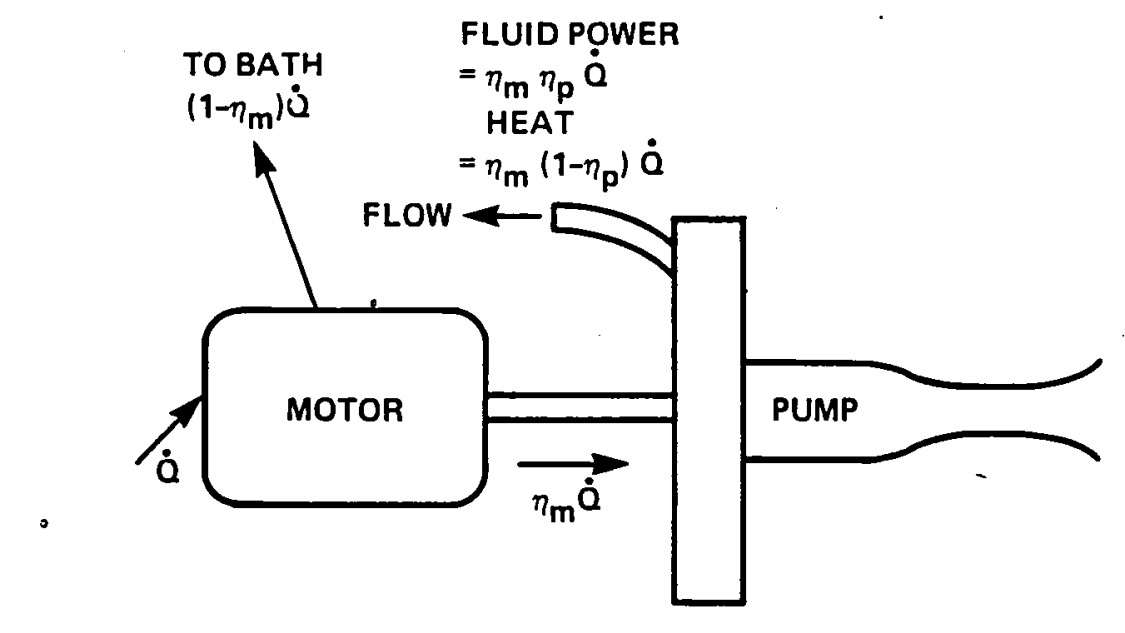

Fig. 2 Power flow in $C P$ where $Q$ is the input power to the motor, $\eta_{m}$ is the efficiency of the motor, and $n_{p}$ is the efficiency of the pump. 


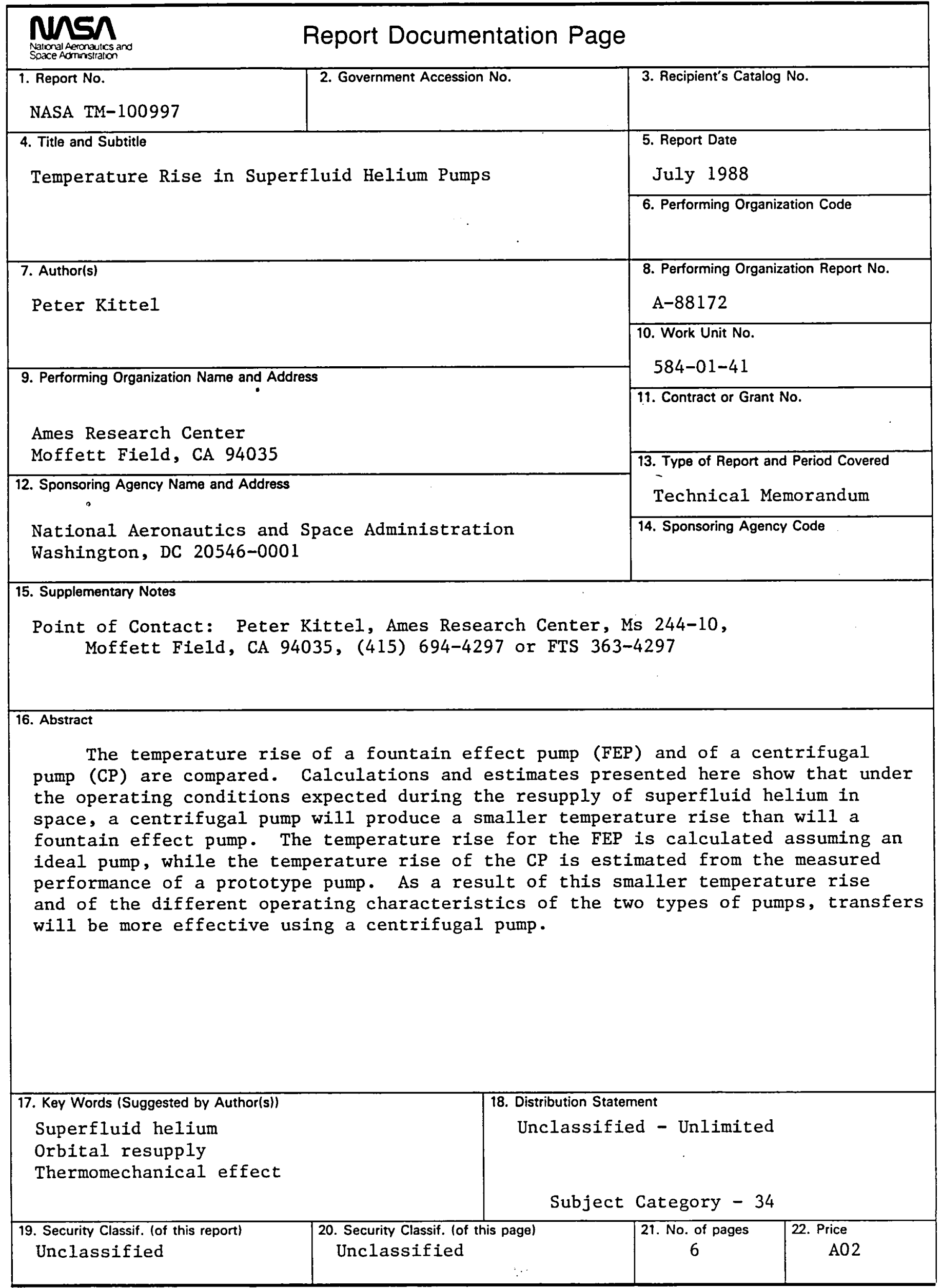

